\title{
The association between platelet indices, cognitive screening tests and functional dependence screening questionnaires in hospitalized older people
}

\author{
Krzysztof Socha $^{1} \cdot$ Elizaveta Fife $^{1} \cdot$ Łukasz Kroc $^{1} \cdot$ Tomasz Kostka $^{1}$
}

Received: 16 January 2019 / Accepted: 18 June 2019 / Published online: 1 July 2019

(c) The Author(s) 2019

\section{Key summary points}

Aim To investigate whether the cognitive or functional status is related to basic platelet indices in hospitalized older adults. Findings After adjustment for multiple confounders, the Mini-Mental State Examination Scale, the seven-point Clock Drawing Test, the Katz ADL scale, the Lawton IADL scale and VES-13 are, except one weak association, not directly related to basic platelet indices measured in the routine complete blood count.

Message The basic platelet indices measured in the routine complete blood count are not consistently associated with the cognitive and functional status evaluated by screening tests. The lack of translation of these associations in clinical use could be due to the multifaceted effect of diseases other than dementia.

\begin{abstract}
Purpose Activated platelets have been suggested to play an important role in the pathogenesis of dementia. Recent studies have shown contradictory results concerning the relationship between blood platelet indices and cognitive performance.

Methods This cross-sectional study evaluates the relationship between basic platelet indices and cognitive and functional performance of 754 men and women, aged 60-105 years old, admitted to the geriatric hospital unit. Assessment of global cognitive function and functional ability were performed using Mini-Mental State Examination, the seven-point Clock Drawing Test, the Katz Activities of Daily Living, the Lawton Instrumental Activities of Daily Living Scale and the Vulnerable Elders Survey Questionnaire. As platelet indices, platelet count, mean platelet volume, plateletcrit and platelet distribution width were measured.

Results There was no significant correlation between cognitive performance and platelet indices. Out of the functional dependence questionnaires, only the Katz Activities of Daily Living and the Vulnerable Elders Survey Questionnaire were weakly correlated with platelet count $(r=-0.080$ and $r=0.096)$ and plateletcrit $(r=-0.075$ and 0.082 , respectively), but these associations diminished in sex-specific analyses.

Conclusions Cognitive and functional status are not systematically related to platelet indices in multimorbid hospitalized older adults.
\end{abstract}

Keywords Platelet count · Mean platelet volume $\cdot$ Plateletcrit $\cdot$ Platelet distribution width $\cdot$ Alzheimer's disease $\cdot$ Vascular dementia

\section{Introduction}

Krzysztof Socha

krzysztof.socha@stud.umed.lodz.pl

1 Department of Geriatrics, Medical University of Lodz, Pieniny 30, 92-115 Lodz, Poland
Due to the high prevalence of dementia, problems of treatment, patients' independence and need for care, treating older patients represents a growing challenge for health systems and social care [1]. There is a notion that the most common causes of dementia, Alzheimer's disease (AD) and vascular dementia (VD), sit on different parts of the 
spectrum of a single syndrome, where vascular risk factors play a vital role $[2,3]$. Blood platelets show similarities to neurons, and previous studies have identified changes in their activity, both in dementia and in many cardiovascular conditions [4, 5]. It is known that blood platelets, like neurons, can metabolize serotonin, amyloid precursor protein and amyloid-beta derivative [6-8]. The changes in platelet activity occurring in old age include lower stimulation thresholds of platelet aggregation, a decrease in the number of plasma membrane receptors for platelet prostacyclin and an increase in the production of fibrinogen activator inhibitor by platelets. All those modifications promote prothrombotic and proinflammatory states [9]. Previous studies indicate that altered platelet activity may occur in response to increased serum concentration of interleukin 6 , secreted primarily by monocytes and macrophages $[10,11]$. Platelet activity can be assessed by simple platelet parameters such as platelet count (PLT), mean platelet volume (MPV) and platelet distribution width (PDW) routinely obtained during analysis of peripheral blood morphology [12-14]. Recent studies showed contradictory results concerning the relationship between MPV and cognitive performance [15-19]. Therefore, the aim of the study was to evaluate the relationship between basic platelet indices (PI) and the cognitive and functional performance of older patients admitted to the hospital ward.

\section{Methods}

\section{Participants}

The cross-sectional study initially included 1237 patients, aged $\geq 60$ years old, hospitalized in the acute care Geriatric University Clinic, Lodz Central Veterans' Hospital (Poland), during a 2-year period. The inclusion criteria comprised the presence of a complete blood count including blood platelet indices, and conducted cognitive and physical ability assessment. The criteria for the participation in this study were also efficient verbal communication and consent given for the participation. Out of the 1237 hospitalization cases, 483 were excluded from the study due to repetitive measurements or incomplete data, usually because of severe dementia or terminal illness. As a result, 754 patients, all of them Caucasian (218 men and 536 women), were included in the study. Of those 754 subjects, 242 were with dementia of various etiologies but were able to complete the questionnaires and were therefore included in the study. The diagnosis of dementia was established based on previous medical records and anamnesis provided by patients' caregivers.

Also, on the basis of an accurate interview with the patient and/or caregiver and a review of previous medical records, we analysed the participants' medical history including a range of pathological conditions that were active in the pre-hospital admission period: hypertension, diabetes, congestive heart disease, ischemic heart disease, atrial fibrillation, previous myocardial infarction or stroke, hypercholesterolemia, chronic pulmonary diseases, osteoporosis, osteoarthrosis, dementia and depression. Anthropometric measurements including body mass and height were obtained. Body mass index (BMI) was calculated as body mass in kilograms divided by the height in metres squared.

The assessment of global cognitive function and functional ability was performed by qualified physicians using the Mini-Mental State Examination (MMSE), the sevenpoint Clock Drawing Test (CDT), the Katz Index of Independence in Activities of Daily Living (ADL), the Lawton Instrumental Activities of Daily Living Scale (IADL) and the Vulnerable Elders Survey (VES-13) questionnaire [20-24].

The study was approved by the Ethics Committee of the Medical University of Lodz and written informed consent was obtained from the participants.

\section{Blood collection and haematological measurements}

The haematology values used in the study were obtained from the first blood collection, taken from participants in the morning after a minimum $8 \mathrm{~h}$ of fasting. Blood samples were obtained by venipuncture in a volume of $3 \mathrm{~mL}$ using blood tubes $\left(\mathrm{K}_{3}\right.$ EDTA) and the Vacutainer BD system and were sent to laboratory directly after collection. Blood samples were delivered to the hospital's central laboratory; samples which were older than $2 \mathrm{~h}$ from collection were excluded. All haematological measurements were performed using an automated Advia 2120 (optical method) analyser by Siemens. The following PI were measured: PLT, MPV, plateletcrit (PCT) and PDW. Serum levels of C-reactive protein (CRP) were determined in fresh blood using latexenhanced immunoturbidimetry.

\section{Statistical analysis}

Statistical analysis was carried out using Statistica 13 software. Power calculation was performed to estimate the required sample size. For a correlation of 0.1 between two variables, the required sample size was found to be 782 , and for a correlation of 0.2 , the required sample size was found to be 193 (with a power of 0.80 and alpha of 0.05). The data were verified for normality of distribution (Kolmogorov-Smirnov test) and equality of variances (Levene test). Mean \pm standard deviation (SD) was used for continuous variables, while relative frequencies were used for categorical variables. The one-way analysis of variance (ANOVA), Mann-Whitney test, and Chi square test with Yates' correction were used to compare the groups. Pearson product 
moment or Spearman correlation coefficients were used to assess the relationship between two quantitative variables. Multiple regression analysis was performed and partial correlations between PI and functional/cognitive assessment were calculated to control the effects of age, BMI and CRP. General linear models and logistic regression models were constructed to identify factors independently predicting functional and cognitive assessment variables with PI, age, sex, BMI, CRP and concomitant diseases, as independent

Table 1 Basic characteristics of the study group

\begin{tabular}{lccl}
\hline & Women $(n=536)$ & Men $(n=218)$ & \\
\hline Age (years) & $80.2 \pm 8.0$ & $82.0 \pm 8.4$ & $p<0.01$ \\
BMI $\left(\mathrm{kg} / \mathrm{m}^{2}\right)$ & $26.0 \pm 4.9$ & $25.8 \pm 3.9$ & NS \\
Haemoglobin $(\mathrm{g} / \mathrm{dL})$ & $12.9 \pm 1.5$ & $13.1 \pm 1.8$ & $\mathrm{NS}$ \\
WBC $\left(\times 10^{9} / \mathrm{L}\right)$ & $7.73 \pm 3.85$ & $7.85 \pm 4.59$ & $\mathrm{NS}$ \\
CRP $(\mathrm{mg} / \mathrm{dl})$ & $16.4 \pm 41.9$ & $20.7 \pm 57.8$ & NS \\
PLT $\left(\times 10^{9} / \mathrm{L}\right)$ & $263.9 \pm 97.7$ & $235.2 \pm 93.9$ & $p<0.001$ \\
MPV (fL) & $8.69 \pm 0.98$ & $8.90 \pm 1.08$ & $p<0.01$ \\
PCT $(\%)$ & $0.22 \pm 0.07$ & $0.20 \pm 0.08$ & $p<0.01$ \\
PDW (\%) & $52.9 \pm 7.6$ & $53.73 \pm 7.65$ & NS \\
MMSE (points) & $23.8 \pm 5.9$ & $23.8 \pm 6.0$ & NS \\
CDT (points) & $3.67 \pm 2.85$ & $3.36 \pm 2.94$ & NS \\
ADL (points) & $4.96 \pm 1.54$ & $4.98 \pm 1.55$ & NS \\
IADL (points) & $5.67 \pm 2.69$ & $5.42 \pm 2.91$ & NS \\
VES-13 (points) & $6.27 \pm 2.70$ & $6.15 \pm 2.67$ & NS \\
\hline
\end{tabular}

$B M I$ body mass index, $W B C$ white blood cell count, $C R P$ C-reactive protein, $P L T$ platelet count, $M P V$ mean platelet volume, $P C T$ plateletcrit, $P D W$ platelet distribution width, MMSE Mini-Mental State Examination, $C D T$ seven-point Clock Drawing Test, $A D L$ Katz Index of Independence in Activities of Daily Living, IADL Lawton Instrumental Activities of Daily Living Scale, VES-13 Vulnerable Elders Survey, NS non-significant. The one -way analysis of variance (ANOVA) and Mann-Whitney test were used to compare the groups variables. ADL ( $\leq 5$ vs. 6 ) and IADL ( $\leq 7$ vs. 8 ) values were dichotomised, while MMSE and CRP values were log transformed for the purpose of multivariate analyses. Values of $p$ below 0.05 were considered statistically significant.

\section{Results}

The general, anthropometric and biochemical characteristics of the patients are presented in Table 1 . Women predominated in the study group ( $71 \%$ women vs. $29 \%$ men) as did the old subgroup, as classified by the WHO (75-89 years old). The mean age of the participants was $80.69 \pm 8.16$ years, with men being almost 2 years older than women. Men had lower PLT and PCT but higher MPV.

Table 2 presents the prevalence of selected concomitant disorders. Men were more likely to have atrial fibrillation and a history of myocardial infarction. Hypercholesterolemia, osteoporosis, osteoarthrosis and depression were more often reported in women (Table 2). Concomitant diseases were related to both PI and cognitive and physical assessment data. Patients with diabetes had higher MPV as compared to the subjects without diabetes $(9.02 \pm 1.09$ vs. $8.67 \pm 0.96 ; p<0.001)$. Subjects with previous myocardial infarction had lower PLT $(227.0 \pm 80.2$ vs. $259.0 \pm 98.8$; $p=0.005)$ and PCT $(0.20 \pm 0.06$ vs. $0.22 \pm 0.08 ; p=0.04)$, with higher MPV $(9.10 \pm 1.07$ vs. $8.71 \pm 1.00 ; p=0.01)$ and PDW (54.7 \pm 7.5 vs. $52.9 \pm 7.6 ; p=0.04)$ as compared to the subjects without previous myocardial infarction. Chronic heart failure was associated with higher MPV $(8.90 \pm 1.09$ vs. $8.63 \pm 0.94 ; p<0.001)$ and PDW $(53.9 \pm 7.8$ vs. $52.5 \pm 7.5 ; p=0.009)$. Subjects with atrial fibrillation had higher PDW $(55.3 \pm 7.6$ vs. $52.6 \pm 7.6 ; p<0.001)$ as compared to subjects without atrial fibrillation. Difference of PLT between patients with hypertension and healthy
Table 2 Prevalence of selected concomitant diseases

\begin{tabular}{llll}
\hline Prevalence (\%) & Women, $n=536$ & Men, $n=218$ & Chi square test \\
\hline Hypertension & 78.0 & 78.9 & NS \\
Diabetes & 21.6 & 26.6 & NS \\
Coronary heart disease & 44.0 & 48.6 & NS \\
Previous myocardial infarction & 8.2 & 16.1 & $p=0.002$ \\
Previous stroke & 14.7 & 16.5 & NS \\
Chronic heart failure & 44.4 & 50.0 & NS \\
Atrial fibrillation & 15.9 & 22.9 & $p=0.028$ \\
Hypercholesterolemia & 44.0 & 31.7 & $p=0.022$ \\
Chronic pulmonary diseases & 14.4 & 16.1 & NS \\
Osteoporosis & 35.3 & 5.5 & $p<0.001$ \\
Osteoarthrosis & 38.6 & 25.2 & $p<0.001$ \\
Depression & 28.5 & 17.4 & $p=0.002$ \\
Dementia & 31.2 & 34.4 & NS \\
\hline
\end{tabular}

$N S$ non-significant. Chi square test with Yates' correction was used to compare the groups 
subjects was of borderline significance $(252.1 \pm 93.6$ vs. $268.4 \pm 109.6$, respectively; $p=0.10$ ), and hypertension was also included in the multivariate analysis. PI was not different between patients diagnosed with dementia and healthy peers.

MMSE and CDT were lower in subjects with coronary heart disease, heart failure, atrial fibrillation, after stroke, with depression and dementia. ADL and IADL were lower in subjects with coronary heart disease, with heart failure, after stroke, with depression and dementia, and were higher in subjects with hypercholesterolemia. VES-13 was higher (worse) in subjects with coronary heart disease, after stroke, with heart failure, with depression and dementia.

In the whole studied population, PLT $(r=-0.12$; $p<0.01)$ and PCT $(r=-0.12 ; p<0.01)$ were inversely related to age and MPV was directly associated with BMI $(r=0.092 ; p<0.05)$. In women, PCT $(r=-0.087 ; p<0.05)$ was inversely related to age and MPV was directly associated with BMI $(r=0.12 ; p<0.01)$. In men, the relationship of age to PCT was of borderline significance $(r=-0.13$; $p=0.06$ ). A direct positive correlation was found between PLT and CRP, and between PCT and CRP (Spearman's $r=0.23, p<0.001$ and $r=0.25, p<0.001$, respectively). CRP was inversely associated with MMSE, CDT, ADL, IADL and directly to VES-13 with the highest correlation coefficient found between ADL and CRP (Spearman's $r=-0.27, p<0.001$ ).

Our results also identified a strong positive correlation of MPV with PDW and a negative correlation between MPV and PLT. PDW was inversely correlated to PLT and PCT, with an obvious strong direct correlation between PLT and PCT. An applied antiplatelet pharmacotherapy involved aspirin (47\%), ticlopidine (0.4\%) and clopidogrel (3.5\% of the subjects). Platelet indices were not related to the applied antiplatelet therapy (aspirin, ticlopidine and clopidogrel merged together for statistical analysis).

\section{The relationship between platelet indices and functional and cognitive assessment}

Spearman's rank correlation coefficients between PI and cognition function (MMSE, CDT) were not statistically significant, neither in the whole study group nor in sex groups. A very weak negative correlation $(p<0.05)$ was revealed between PLT and PCT with ADL $(r=-0.087$ and $r=-0.077$ ), but these correlations became insignificant when subjected to sex-specific analyses. Observed correlations were quasi-identical when analysing per age categories: in subjects aged $<80$ years $(n=288)$ and aged $\geq 80$ years $(n=466)$ and in subjects aged $<85$ years $(n=483)$ and $\geq 85$ years $(n=271)$. Association of PI to functional and cognitive assessment data was similar in subjects with MMSE $\geq 11(n=728)$ and subjects with MMSE $\geq 24$ $(n=482)$ as compared to the whole studied population. The only weak correlations of ADL to PLT and PCT with Spearman's $r$ ranging from -0.089 to -0.10 were found.

Table 3 presents partial correlations (partial $r$ ) between platelet indices and functional and cognitive assessment in the whole study group and according to sex after adjustment for age, BMI and CRP. Partial correlation coefficients between PI and cognition function (MMSE, CDT) were not statistically significant, neither in the whole study group nor in sex groups. A very weak negative correlation $(p<0.05)$ between PLT and PCT with ADL, and positive correlation between PLT and PCT with VES-13 was revealed, but these correlations became insignificant when subjected to sex-specific analyses. PDW was also inversely related to VES-13 in men (Table 3).

Multivariate analyses with adjustments for age, sex, BMI, CRP and concomitant diseases with significant relationship to PI or functional and cognitive assessment variables in bivariate associations have also been performed. From PI, only PLT emerged as independent predictor of ADL. Age, $\mathrm{CRP}$, coronary heart disease and dementia were independent predictors of MMSE. CDT was determined by age, CRP, depression and dementia. PLT (with $p=0.013$ ) together with age, CRP, history of previous stroke, depression and

Table 3 Partial correlations (partial $r$ ) between platelet indices and functional and cognitive assessment in the whole study group and according to sex after adjustment for age, BMI and CRP

\begin{tabular}{|c|c|c|c|c|c|}
\hline & MMSE & CDT & ADL & IADL & VES-13 \\
\hline \multicolumn{6}{|c|}{ All participants $(n=754)$} \\
\hline PLT & -0.01 & -0.04 & $-0.08^{*}$ & -0.03 & $0.096 * *$ \\
\hline MPV & 0.03 & 0.04 & 0.05 & -0.01 & -0.05 \\
\hline PCT & 0.007 & -0.01 & $-0.075 *$ & -0.02 & $0.082^{*}$ \\
\hline PDW & -0.01 & 0.05 & 0.04 & 0.05 & -0.067 \\
\hline \multicolumn{6}{|c|}{ Women $(n=536)$} \\
\hline PLT & -0.03 & -0.01 & -0.05 & 0.003 & 0.082 \\
\hline MPV & 0.02 & 0.01 & 0.01 & -0.06 & 0.006 \\
\hline PCT & -0.03 & -0.003 & -0.04 & -0.008 & 0.084 \\
\hline PDW & -0.02 & 0.056 & 0.04 & 0.02 & -0.02 \\
\hline \multicolumn{6}{|c|}{ Men $(n=218)$} \\
\hline PLT & -0.02 & -0.03 & -0.06 & -0.06 & 0.05 \\
\hline MPV & 0.056 & 0.061 & 0.06 & 0.073 & -0.12 \\
\hline PCT & 0.058 & 0.007 & -0.001 & -0.098 & 0.02 \\
\hline PDW & 0.005 & 0.008 & 0.085 & 0.088 & $-0.15^{*}$ \\
\hline
\end{tabular}

${ }^{*} p<0.05 ; * * p<0.01$

PLT platelet count, MPV mean platelet volume, $P C T$ plateletcrit, $P D W$ platelet distribution width, MMSE Mini-Mental State Examination, $C D T$ seven-point Clock Drawing Test, $A D L$ Katz Index of Independence in Activities of Daily Living, IADL Lawton Instrumental Activities of Daily Living Scale, VES-13 Vulnerable Elders Survey. Multiple regression analysis was performed and partial correlations between PI and functional/cognitive assessment were calculated to control the effects of age, BMI and CRP 
dementia were independent predictors of ADL. IADL was determined by age, CRP, history of previous stroke, depression and dementia. VES-13 was predicted by age, CRP, depression and dementia.

\section{Discussion}

The present study evaluates the relationship between basic PI and cognitive and functional performance in the largest population of older hospitalized patients studied so far. Observed sex-specific differences in PI data and relationship of PI to age and BMI are generally similar to that found in previous studies [25-30].

A number of studies published in recent decades suggest that platelet function in $\mathrm{AD}$ may be altered $[31,32]$. This influence of platelet activation on pathophysiological process in $\mathrm{AD}$ has been suggested in many studies [5, 33-36]. AD patients were reported to have increased levels of MPV and decreased levels of PDW [37]. MPV was higher in patients with acute ischemic stroke as compared to age- and sex-matched controls [38]. MPV has been proposed to be a novel index for silent cerebral infarction regardless of classical cardiovascular risk factors [39]. In the present study, no correlation was found between PLT, MPV, PCT or PDW and MMSE or CDT score in a large population of hospitalized older adults. Previous data suggested the presence of differences in MPV and PDW in patients with $\mathrm{AD}$ as compared to those with mild cognitive impairment (MCI), as well as between MCI group and a group of healthy controls, with a strong positive correlation between MPV and MMSE $(r=0.576, p<0.001)$ and PDW and MMSE $(r=0.465, p<0.001)$ after adjusting for age, sex, and body mass index [17]. Additionally, a study evaluating the relationship between PI and type of dementia (VD or AD) identified a positive correlation between MPV, PDW and MMSE, and found a lower PDW in the AD group than the VD group [15]. Conflicting results were provided by Turkish researchers, who found that AD patients had higher mean MPV than control group without cognitive dysfunction $[16,19]$. Our results concerning the lack of coincidence of PI and MMSE are consistent with those of Koçer et al. who found no difference in PI between outpatients with AD and healthy controls [18]. Likewise, Koç et al. reported that MPV did not differentiate AD patients with mild and moderate stage based on MMSE scoring [19].

There are relatively few studies published to date, which examine the relationship between PI and functional dependence [40-42]. Conflicting data are also available on the association of PI to prognosis in cancer patients or in patients with coronary artery disease [41-43]. There was no correlation between the severity of functional ability assessed by the Rankin scale and the MPV in patients with recent ischemic stroke [38]. Platelet morphology contributes to platelet reactivity, but those associations are complex and far from complete understanding [44]. Interestingly, our results identify a significant negative correlation between Katz' Index with PLT and PCT and positive correlation between PLT and PCT with VES-13, although these effects are very weak and not evident in separate sex analyses. In addition, no relationship was found between the results of IADL and PI. Furthermore, in fully adjusted models, only PLT emerged as a weak independent predictor of ADL. All other cognitive and functional status measures were determined by age, CRP and concomitant diseases. Our study was carried out as a part of a comprehensive geriatric evaluation at a particular moment for the patient - at the beginning of hospitalization in the geriatric ward. De Gonzalo-Calvo et al. examined a group of 119 patients in a nursing home setting who were around 5 years older, more functionally dependent (the Katz ADL $2 \pm 2$ vs. $5 \pm 1.5$ ) but with fewer medical diagnoses ( $2 \pm 1$ vs. $3 \pm 1.5$ ); they also found no relationship between PI and the Katz or Barthel indices, but they found that low levels of PDW were associated with increased 1-year mortality [40]. Therefore, as observed in the present report, the negative association of VES-13 and PDW in men seems worth confirming in future studies.

The observed correlations between PI and ADL or VES13 could be influenced by the high prevalence of patients with acute infection and inflammation. The infectiondependent changes in platelet properties are well described [45]. A strong direct positive correlation between PLT and CRP and between PCT and CRP was found in the present study, together with characteristic correlations between PI. These associations are already known as they accompany platelet activation [29, 46]. CRP was also an independent predictor of all cognitive and functional tests in multivariate analysis. Although we have adjusted for CRP in multivariate assessments, obtained data may be specific for multimorbid older population with frequent inflammation status.

\section{Conclusions}

Hospitalized adults aged 60 years and older demonstrated no associations between PI and cognitive screening test (MMSE and CDT). Significant but weak correlations were found between PI and the results of the functional dependence screening questionnaires, but only between the ADL and VES-13 with PLT and PCT. In addition, these correlations except one were not evident in fully adjusted multivariate models. These associations could be influenced by the high prevalence of patients with acute infection and inflammation and multiple coexisting diseases. Therefore, platelet indices are not consistently associated with the cognitive 
and functional performance of older patients admitted to the hospital ward.

Acknowledgements This study was supported by Grant 503/6-07701/503-61-001 from the Medical University of Lodz.

Funding This study was supported by Grant 503/6-077-01/503-61-001 from the Medical University of Lodz.

\section{Compliance with ethical standards}

Conflict of interest On behalf of all authors, the corresponding author states that there is no conflict of interest.

Ethical approval All procedures performed in studies involving human participants were in accordance with the ethical standards of the institutional research committee: the Ethics Committee of the Medical University of Lodz, and with the 1964 Helsinki Declaration and its later amendments or comparable ethical standards.

Informed consent Informed consent was obtained from all individual participants included in the study.

Open Access This article is distributed under the terms of the Creative Commons Attribution 4.0 International License (http://creativeco mmons.org/licenses/by/4.0/), which permits unrestricted use, distribution, and reproduction in any medium, provided you give appropriate credit to the original author(s) and the source, provide a link to the Creative Commons license, and indicate if changes were made.

\section{References}

1. World Health Organization (2012) Dementia: a public health priority. WHO, Geneva. https://apps.who.int/iris/bitstream/handl e/10665/75263/9789241564458_eng.pdf. Accessed 15 Mar 2017

2. de la Torre JC (2004) Is Alzheimer's disease a neurodegenerative or a vascular disorder? Data, dogma, and dialectics. Lancet Neurol 3(3):184-190

3. Alagiakrishnan K, McCracken P, Feldman H (2006) Treating vascular risk factors and maintaining vascular health: is this the way towards successful cognitive ageing and preventing cognitive decline? Postgrad Med J 82(964):101-105

4. Stellos K, Katsiki N, Tatsidou P, Bigalke B, Laske C (2014) Association of platelet activation with vascular cognitive impairment: implications in dementia development? Curr Vasc Pharmacol 12(1):152-154

5. Laske C, Sopova K, Stellos K (2012) Platelet activation in Alzheimer's disease: from pathophysiology to clinical value. Curr Vasc Pharmacol 10(5):626-630

6. Vignini A, Morganti S, Salvolini E, Sartini D, Luzzi S, Fiorini R, Provinciali L, Di Primio R, Mazzanti L, Emanuelli M (2013) Amyloid precursor protein expression is enhanced in human platelets from subjects with Alzheimer's disease and frontotemporal lobar degeneration: a real-time PCR study. Exp Gerontol 48(12):1505-1508

7. Chen M, Inestrosa NC, Ross GS, Fernandez HL (1995) Platelets are the primary source of amyloid beta-peptide in human blood. Biochem Biophys Res Commun 213(1):96-103

8. Muck-Seler D, Presecki P, Mimica N, Mustapic M, Pivac N, Babic A, Nedic G, Folnegovic-Smalc V (2009) Platelet serotonin concentration and monoamine oxidase type B activity in female patients in early, middle and late phase of Alzheimer's disease. Prog Neuropsychopharmacol Biol Psychiatry 33(7):1226-1231

9. Mohebali D, Kaplan D, Carlisle M, Supiano MA, Rondina MT (2014) Alterations in platelet function during aging: clinical correlations with thromboinflammatory disease in older adults. J Am Geriatr Soc 62(3):529-535

10. Oleksowicz L, Mrowiec Z, Isaacs R, Dutcher JP, Puszkin E (1995) Morphologic and ultrastructural evidence of interleukin-6 induced platelet activation. Am J Hematol 48(2):92-99

11. Zuliani G, Ranzini M, Guerra G, Rossi L, Munari MR, Zurlo A, Volpato S, Atti AR, Blè A, Fellin R (2007) Plasma cytokines profile in older subjects with late onset Alzheimer's disease or vascular dementia. J Psychiatr Res 41(8):686-693

12. Tsiara S, Elisaf M, Jagroop IA, Mikhailidis DP (2003) Platelets as predictors of vascular risk: is there a practical index of platelet activity? Clin Appl Thromb Hemost 9(3):177-190

13. Kang SJ, Park BJ, Shim JY, Lee HR, Hong JM, Lee YJ (2012) Mean platelet volume (MPV) is associated with leukoaraiosis in the apparently healthy elderly. Arch Gerontol Geriatr 54(2):e118-e121

14. Vagdatli E, Gounari E, Lazaridou E, Katsibourlia E, Tsikopoulou F, Labrianou I (2010) Platelet distribution width: a simple, practical and specific marker of activation of coagulation. Hippokratia 14(1):28-32

15. Liang QC, Jin D, Li Y, Wang RT (2014) Mean platelet volume and platelet distribution width in vascular dementia and Alzheimer's disease. Platelets 25(6):433-438

16. Yesil Y, Kuyumcu ME, Cankurtaran M, Uz B, Kara A, Kilic MK, Halil M, Ulger Z, Yavuz BB, Haznedaroğlu IC, Ariogul $S$ (2012) Increased mean platelet volume (MPV) indicating the vascular risk in Alzheimer's disease (AD). Arch Gerontol Geriatr 55(2):257-260

17. Wang RT, Jin D, Li Y, Liang QC (2013) Decreased mean platelet volume and platelet distribution width are associated with mild cognitive impairment and Alzheimer's disease. J Psychiatr Res 47(5):644-649

18. Koçer A, Yaman A, Niftaliyev E, Dürüyen H, Eryılmaz M, Koçer E (2013) Assessment of platelet indices in patients with neurodegenerative diseases: mean platelet volume was increased in patients with Parkinson's disease. Curr Gerontol Geriatr Res 2013:986254

19. Koç ER, Uzar E, Çirak Y, Parlak Demir Y, Ilhan A (2014) The increase of mean platelet volume in patients with Alzheimer disease. Turk J Med Sci 44(6):1060-1066

20. Folstein MF, Folstein SE, McHugh PR (1975) "Mini-mental state." A practical method for grading the cognitive state of patients for the clinician. J Psychiatr Res 12(3):189-198

21. Watson YI, Arfken CL, Brige SJ (1993) Clock completion: an objective screening test for dementia. J Am Geriatr Soc 41(11):1235-1240

22. Katz S, Ford AB, Moskowitz RW, Jackson BA, Jaffe MW (1963) Studies of illness in the aged. The index of ADL: a standardized measure of biological and psychological function. JAMA 185:914-919

23. Lawton MP, Brody EM (1969) Assessment of older people: selfmaintaining and instrumental activities of daily living. Gerontologist 9(3):179-186

24. Saliba D, Elliott M, Rubenstein LZ, Solomon DH, Young RT, Kamberg CJ, Roth C, MacLean CH, Shekelle PG, Sloss EM, Wenger NS (2001) The vulnerable elders survey: a tool for identifying vulnerable older people in the community. J Am Geriatr Soc 49(12):1691-1699

25. Ruocco L, Del Corso L, Romanelli AM, Deri D, Pentimone F (2001) New hematological indices in the healthy elderly. Minerva Med 92(2):69-73 
26. Tsang CW, Lazarus R, Smith M, Mitchell P, Koutts J, Burnett L (1998) Hematological indices in an older population sample: derivation of healthy reference values. Clin Chem 44(1):96-101

27. Butkiewicz AM, Kemona H, Dymicka-Piekarska V, MatowickaKarna J, Radziwon P, Lipska A (2006) Platelet count, mean platelet volume and thrombocytopoietic indices in healthy women and men. Thromb Res 118(2):199-204

28. Balduini CL, Noris P (2014) Platelet count and aging. Haematologica 99(6):953-955

29. Verdoia M, Schaffer A, Barbieri L, Bellomo G, Marino P, Sinigaglia F, Suryapranata H, De Luca G, Novara Atherosclerosis Study (NAS) group (2015) Impact of age on mean platelet volume and its relationship with coronary artery disease: a single-centre cohort study. Exp Gerontol 62:32-36

30. Han S, Gan D, Wang G, Ru Y, Huang C, Lin J, Zhang L, Meng Z, Zhu S (2018) Associations of platelet indices with body fat mass and fat distribution. Obesity (Silver Spring) 26(10):1637-1643

31. Johnston JA, Liu WW, Coulson DT, Todd S, Murphy S, Brennan S, Foy CJ, Craig D, Irvine GB, Passmore AP (2008) Platelet betasecretase activity is increased in Alzheimer's disease. Neurobiol Aging 29(5):661-668

32. Colciaghi F, Marcello E, Borroni B, Zimmermann M, Caltagirone C, Cattabeni F, Padovani A, Di Luca M (2004) Platelet APP, ADAM 10 and BACE alterations in the early stages of Alzheimer disease. Neurology 62(3):498-501

33. Stellos K, Panagiota V, Kögel A, Leyhe T, Gawaz M, Laske C (2010) Predictive value of platelet activation for the rate of cognitive decline in Alzheimer's disease patients. J Cereb Blood Flow Metab 30(11):1817-1820

34. Catricala S, Torti M, Ricevuti G (2012) Alzheimer disease and platelets: how's that relevant. Immun Ageing 9(1):20

35. Casoli T, Di Stefano G, Balietti M, Solazzi M, Giorgetti B, Fattoretti P (2010) Peripheral inflammatory biomarkers of Alzheimer's disease: the role of platelets. Biogerontology 11(5):627-633

36. Sevush S, Jy W, Horstman LL, Mao WW, Kolodny L, Ahn YS (1998) Platelet activation in Alzheimer disease. Arch Neurol 55(4):530-536

37. Chen SH, Bu XL, Jin WS, Shen LL, Wang J, Zhuang ZQ, Zhang T, Zeng F, Yao XQ, Zhou HD, Wang YJ (2017) Altered peripheral profile of blood cells in Alzheimer disease A hospital-based casecontrol study. Medicine (Baltimore) 96(21):e6843

38. Neki NS, Minda N, Jain A (2016) A study of association of mean platelet volume and ischaemic stroke. Asian Pac J Health Sci 3(4):212-219
39. Li B, Liu X, Cao ZG, Li Y, Liu TM, Wang RT (2014) Elevated mean platelet volume is associated with silent cerebral infarction. Intern Med J 44(7):653-657

40. de Gonzalo-Calvo D, de Luxán-Delgado B, Rodríguez-González S, García-Macia M, Suárez FM, Solano JJ, Rodríguez-Colunga MJ, Coto-Montes A (2013) Platelet distribution width is associated with 1-year all-cause mortality in the elderly population. J Clin Gerontol Geriatr 4(1):12-16

41. Pyo JS, Sohn JH, Kang G (2016) Diagnostic and prognostic roles of the mean platelet volume in malignant tumors: a systematic review and meta-analysis. Platelets 27(8):722-728

42. Omar M, Tanriverdi O, Cokmert S, Oktay E, Yersal O, Pilancı KN, Menekse S, Kocar M, Sen CA, Ordu C, Goksel G, Meydan N, Barutca S, Turkish Descriptive Oncological Researches Group (2018) Role of increased mean platelet volume (MPV) and decreased MPV/platelet count ratio as poor prognostic factors in lung cancer. Clin Respir J 12(3):922-929

43. Sansanayudh N, Numthavaj P, Muntham D, Yamwong S, McEvoy M, Attia J, Sritara P, Thakkinstian A (2015) Prognostic effect of mean platelet volume in patients with coronary artery disease. A systematic review and meta-analysis. Thromb Haemost 114(6):1299-1309

44. Karolczak K, Soltysik B, Kostka T, Witas PJ, Watala C (2019) Platelet and red blood cell counts, as well as the concentrations of uric acid, but not homocysteinaemia or oxidative stress, contribute mostly to platelet reactivity in older adults. Oxid Med Cell Longev 2019:9467562

45. Zareifar S, Farahmand Far MR, Golfeshan F, Cohan N (2014) Changes in platelet count and mean platelet volume during infectious and inflammatory disease and their correlation with ESR and CRP. J Clin Lab Anal 28(3):245-248

46. Demirin H, Ozhan H, Ucgun T, Celer A, Bulur S, Cil H, Gunes C, Yildirim HA (2011) Normal range of mean platelet volume in healthy subjects: insight from a large epidemiologic study. Thromb Res 128(4):358-360

Publisher's Note Springer Nature remains neutral with regard to jurisdictional claims in published maps and institutional affiliations. 